\title{
Re-evaluation of low intensity pulsed ultrasound in treatment of tibial fractures (TRUST): randomized clinical trial
}

\author{
Jason W Busse, ${ }^{1,2,3}$ Mohit Bhandari, ${ }^{1,4}$ Thomas A Einhorn, ${ }^{5}$ Emil Schemitsch, ${ }^{6}$ James D Heckman, ${ }^{7}$ \\ Paul Tornetta, III ${ }^{5}$ Kwok-Sui Leung, ${ }^{8}$ Diane Heels-Ansdell, ${ }^{1}$ Sun Makosso-Kallyth, ${ }^{2}$ \\ Gregory I Della Rocca, ${ }^{9}$ Clifford B Jones, ${ }^{10}$ Gordon H Guyatt ${ }^{1,11}$
}

${ }^{1}$ Department of Clinical

Epidemiology and Biostatistics,

McMaster University, Hamilton,

ON, L8S 4K1, Canada

${ }^{2}$ Department of Anesthesia,

McMaster University, Hamilton,

ON L8S 4K1, Canada

${ }^{3}$ Michael G. DeGroote Institute

for Pain Research and Care,

McMaster University, Hamilton,

ON L8S 4K1, Canada

${ }^{4}$ Department of Surgery,

McMaster University, Hamilton,

ON L8S 4L8, Canada

${ }^{5}$ Department of Orthopedic

Surgery, NYU Langone Medical

Center, New York, NY 10016, USA

${ }^{6}$ Department of Surgery,

University of Western Ontario,

London, ON N6A 4V2, Canada

${ }^{7}$ Department of Orthopedic

Surgery, Dartmouth-Hitchcock

Medical Center, Lebanon, NH

03766, USA

${ }^{8}$ Department of Orthopedics and Traumatology, Chinese University of Hong Kong, Shatin,

Hong Kong, China

${ }^{9}$ Department of Orthopedic

Surgery, University of Missouri,

Columbia, MO 65212, USA

${ }^{10} \mathrm{Center}$ for Orthopedic

Research and Education, CORE,

Banner University, Phoenix, AZ,

85023, USA

${ }^{11}$ Department of Medicine,

McMaster University, Hamilton,

ON L8S 4K1, Canada

Correspondence to: J W Busse, 1200 Main Street West, HSC-2U1,

Department of Anesthesia,

McMaster University, Hamilton,

ON, L8S 4K1, Canada

bussejw@mcmaster.ca

Additional material is published online only. To view please visit

the journal online.

Cite this as: BMJ 2016;355:i5351 http://dx.doi.org/10.1136/bmj.i5351

\section{ABSTRACT}

\section{OBJECTIVE}

To determine whether low intensity pulsed ultrasound (LIPUS), compared with sham treatment, accelerates functional recovery and radiographic healing in patients with operatively managed tibial fractures.

\section{DESIGN}

A concealed, randomized, blinded, sham controlled clinical trial with a parallel group design of 501 patients, enrolled between October 2008 and September 2012, and followed for one year.

SETTING

43 North American academic trauma centers.

\section{PARTICIPANTS}

Skeletally mature men or women with an open or closed tibial fracture amenable to intramedullary nail fixation. Exclusions comprised pilon fractures, tibial shaft fractures that extended into the joint and required reduction, pathological fractures, bilateral tibial fractures, segmental fractures, spiral fractures $>7.5 \mathrm{~cm}$ in length, concomitant injuries that were likely to impair function for at least as long as the patient's tibial fracture, and tibial fractures that showed $<25 \%$ cortical contact and $>1 \mathrm{~cm}$ gap after surgical fixation. 3105 consecutive patients who underwent intramedullary nailing for tibial fracture were assessed, 599 were eligible and 501 provided informed consent and were enrolled.

\section{INTERVENTIONS}

Patients were allocated centrally to self administer daily LIPUS $(n=250)$ or use a sham device $(n=251)$ until their tibial fracture showed radiographic healing or until one year after intramedullary fixation.

\section{MAIN OUTCOME MEASURES}

Primary registry specified outcome was time to radiographic healing within one year of fixation; secondary outcome was rate of non-union. Additional protocol specified outcomes included short form-36 (SF-36) physical component summary (PCS) scores,

\section{WHAT IS ALREADY KNOWN ON THIS TOPIC}

Low intensity pulsed ultrasound (LIPUS) is commonly used to promote fracture healing in North America

The effectiveness this treatment remains uncertain because of limitations of previous trials, including a focus on radiographic fracture healing, which is a surrogate for functional recovery

\section{WHAT THIS STUDY ADDS}

Addition of LIPUS to usual care for patients with fracture failed to accelerate radiographic healing or improve function

return to work, return to household activities, return to $\geq 80 \%$ of function before injury, return to leisure activities, time to full weight bearing, scores on the health utilities index (mark 3), and adverse events related to the device.

\section{RESULTS}

SF-36 PCS data were acquired from 481/501 (96\%) patients, for whom we had 2303/2886 (80\%) observations, and radiographic healing data were acquired from 482/501 (96\%) patients, of whom 82 were censored. Results showed no impact on SF-36 PCS scores between LIPUS and control groups (mean difference $0.55,95 \%$ confidence interval -0.75 to $1.84 ; \mathrm{P}=0.41$ ) or for the interaction between time and treatment $(P=0.30)$; minimal important difference is 3-5 points) or in other functional measures. There was also no difference in time to radiographic healing (hazard ratio 1.07, $95 \%$ confidence interval 0.86 to $1.34 ; \mathrm{P}=0.55$ ). There were no differences in safety outcomes between treatment groups. Patient compliance was moderate; $73 \%$ of patients administered $\geq 50 \%$ of all recommended treatments.

\section{CONCLUSIONS}

Postoperative use of LIPUS after tibial fracture fixation does not accelerate radiographic healing and fails to improve functional recovery.

\section{STUDY REGISTRATION}

ClinicalTrialGov Identifier: NCT00667849.

\section{Introduction}

Tibial shaft fractures, the most common long bone fracture, ${ }^{1}$ are typically managed with intramedullary nailing. ${ }^{2}$ Operatively managed tibial fractures generally require three to six months before patients return to their functional status before injury. Moreover, the limited soft tissue envelope surrounding the bone predisposes tibial fractures to non-union. ${ }^{3}$ Low intensity pulsed ultrasound (LIPUS) is commonly used in North America after fracture surgery to accelerate fracture healing and prevent non-union. ${ }^{24}$ Although randomized trials have suggested that LIPUS improves radiographic healing, inferences are limited because of small sample size, risk of bias, and inconsistent results. ${ }^{5}$ Moreover, radiographic fracture healing represents a surrogate outcome that might not translate into accelerated functional recovery. ${ }^{6}$

In 2004, global revenues for bone stimulators were about $\$ 400 \mathrm{~m}$ (£234 m, €267 m). ${ }^{7}$ Widespread use, however, does not assure effectiveness. Each year, unnecessary interventions are estimated to account for $10-30 \%$ 
of spending on healthcare in the US, or $\$ 250 \mathrm{bn}-\$ 800 \mathrm{bn}$ ( $€ 154$ bn- $€ 490$ bn; €190 bn-€610 bn). ${ }^{8}$ Reasons for unnecessary treatment include knowledge gaps, biased research, profit seeking, patient demand, and rapid uptake of unproved technology..$^{9}$ Interest in curtailing unnecessary treatment is growing. For instance, the American Board of Internal Medicine launched its Choosing Wisely campaign in December 2011, and the BMJ's “Too Much Medicine” initiative highlights the waste of resources on unnecessary care. ${ }^{1011}$

To resolve the uncertainty regarding the role of LIPUS in operatively managed patients with tibial fractures, we conducted a multicentre, sham controlled, randomized controlled trial that prioritized functional recovery. The design of our trial was informed by a pilot study ${ }^{12}$ and a survey of 450 orthopedic trauma surgeons ${ }^{2}$ that found surgeons were managing almost all tibial fractures operatively and using intramedullary nailing in more than $80 \%$ of fractures. Of the respondents, $45 \%$ used bone stimulators-evenly split between LIPUS and electrical stimulation. Our null hypothesis was that there would be no difference in functional recovery or radiographic healing after surgical repair of a traumatic tibial fracture with intramedullary nailing, whether managed with adjunctive LIPUS or a sham device.

\section{Methods}

Trial design

This multicenter, randomized, blinded, sham controlled, parallel group clinical trial was conducted at 43 North American university affiliated academic trauma centres between October 2008 and March 2013. There were no shared patients between the TRUST pilot study ${ }^{12}$ and the TRUST definitive trial.

\section{Patient selection}

Eligible patients were skeletally mature men or women with an open (Gustilo type I-IIIB) or closed (Tscherne grade $0-3$ ) tibial fracture amenable to intramedullary nail fixation who provided written informed consent within 14 days of intramedullary nailing and were willing and able to comply with the study protocol.

We excluded patients in whom wound care precluded ultrasound-skin contact, pilon fractures, tibial shaft fractures that extended into the knee or ankle joint and required reduction, pathological fractures, bilateral tibial fractures, segmental fractures, spiral fractures $>7.5 \mathrm{~cm}$ in length, concomitant injuries that, in the opinion of the attending surgeon, were likely to impair function for at least as long as the patient's tibial fracture, and tibial fractures that showed less than $25 \%$ cortical contact and $>1 \mathrm{~cm}$ gap after intramedullary nail fixation. We also excluded patients if there were likely to be problems with maintaining follow-up (such as no fixed address), patients with cognitive impairment or language difficulties that would impede the valid completion of questionnaires, women who were pregnant or nursing or planned to become pregnant during their treatment period, or patients with osteobiologic implants at the site of their tibial fracture or with active implanted devices such as cardiac pacemakers.

\section{Randomization and masking}

The manufacturer labeled and shipped visually identical active and inactivated (sham) Exogen 4000+ ultrasound device units to investigational sites according to a computer generated randomization plan created by the McMaster University methods center. Randomization was stratified by center and severity of soft tissue injury (open or closed) with randomly varied block sizes ranging from 2 to 4 . After fracture fixation with an intramedullary nail, participating investigators randomized patients, in a 1:1 ratio, to LIPUS or a deactivated device (identical in appearance and apparent function) by accessing a 24 hour toll-free remote telephone randomization system that ensured concealment. Patients, surgeons and other clinicians, data collectors, outcome adjudicators, data analysts, and the industry sponsor were blind to treatment allocation until the data analysis was complete.

\section{Perioperative care}

Trauma fellowship-trained orthopedic surgeons administered standardized care before and after surgery. The type of intramedullary nailing (reamed or unreamed), and the number of interlocking screws used, were at the discretion of the attending surgeon. For closed fractures, the protocol specified preoperative antibiotic administration be continued for 24 hours postoperatively, and for open fractures, antibiotic administration (including a cephalosporin and an aminoglycoside, if indicated for grade IIIA-IIIB fractures) continued for 72 hours postoperatively. Irrigation and debridement of soft tissues and contaminated bone was repeated as necessary, and delayed wound closure, split thickness skin grafting, or muscle flaps (for grade IIIB only) occurred only after the initial surgery. For both open and closed fractures, cortical contact of the fracture ends guided weight bearing. If cortical contact was achieved, patients were instructed to weight bear as tolerated. Otherwise, patients were instructed to partially weight bear on the affected limb until performance of a definitive procedure to achieve contact.

\section{Interventions}

Each patient received a LIPUS device containing a timer that monitored treatment times and automatically turned the unit off after 20 minutes, verbal instructions in its use, and a booklet containing detailed instructions. The device transmits a low intensity ultrasound signal $\left(30 \mathrm{~mW} / \mathrm{cm}^{2}\right)$ to the fracture site through coupling gel. This is similar to diagnostic ultrasound levels used in sonography (fetal monitoring) procedures. Because of the low intensity, patients feel no sensation during treatment. The active and sham devices had the same visual, tactile, and auditory signals and were therefore indistinguishable. Patients self administered treatment once daily for 20 minutes until their surgeon determined that their fracture showed radiographic evidence of bridging at all four cortices or until the 52 week follow-up visit, whichever occurred first. 


\section{Outcome measures}

We assessed outcomes at discharge and at follow-up visits at $6,12,18,26,38$, and 52 weeks postoperatively. We submitted our trial protocol to the US Food and Drug Administration (FDA) for approval with a primary effectiveness outcome of return to function (short form-36 (SF-36) physical component summary (PCS) scores). The FDA, however, requested we change our primary outcome to radiographic healing. After discussion, the FDA agreed to accept a co-primary outcome of SF-36 PCS scores and time to radiographic healing, with the understanding that, in independent analyses, both endpoints had to show a clinically important treatment effect for the results to be considered positive. The primary safety outcome was the difference between treatment groups in the proportion of patients with adverse events related to the device and unplanned secondary procedures related to bone healing and infection.

In December 2015 the industry sponsor edited the trial registry data to reflect the co-primary outcome agreed with the FDA (that is, added SF-36 PCS scores) and replaced the secondary outcome of rate of nonunion with treatment compliance. The primary and secondary outcomes that we have reported are consistent with the original protocol with the modification requested by the FDA.

The SF-36 has shown construct validity, test-retest reliability, and acceptable responsiveness in orthopedic populations; evidence suggests that patients consider a difference of 3-5 points or more as important. ${ }^{13-16}$ Our survey of orthopedic trauma surgeons found that $80 \%$ of respondents thought a reduction in healing time of six weeks or more, attributed to a bone stimulator, would be important to patients. ${ }^{2}$

A central adjudication committee, comprising orthopedic trauma surgeons who were blind to device allocation, independently adjudicated patient eligibility, time to radiographic healing (bridging of three cortices), non-union, secondary procedures, and adverse events related to the fracture. Radiographic non-union was defined as failure of the fracture to progress further towards radiographic healing for at least eight consecutive weeks, after a minimum of six months (26 weeks) after initial intramedullary nailing. Any disagreements were resolved through discussion.

Study centers sent digital photographs of anteroposterior and lateral radiographs to the methods center. The adjudication committee assessed radiographic healing using the radiographic union scale for tibial fractures (RUST) system, which assigns a score to anteroposterior and lateral radiographs based on the assessment of healing at each of the four cortices visible on these projections..$^{17}$ Each cortex receives a score of 1 point if it is deemed to have a fracture line with no callus, 2 points if there is callus present but a fracture line is still visible, and 3 points if there is bridging callus with no evidence of a fracture line. The individual cortical scores were added to give a total for each set of films with 4 being the minimum, indicating the fracture is definitely not healed, and 12 the maximum score, indicating the fracture is fully healed. A cortex was judged as bridged when it achieved a RUST score of 2 or 3 . Although they were deemed radiographically healed when, in the opinion of the adjudication committee, three cortices were bridged, to guard against missing data from misclassification by site investigators, patients continued to use their study device and undergo radiographic evaluation until the site investigator determined that all four cortices were bridged. Anteroposterior and lateral radiographs were standardized, whenever possible, with use of the same $\mathrm{x}$ ray machine at each site and the same exposure settings.

Secondary outcomes were return to work without limitations among those who were employed before their injury; return to household activities without limitations; return to at least $80 \%$ of function from before injury; return to leisure activities without limitations; time to full weight bearing; and scores on the health utilities index (mark 3), which is a validated generic utility measure-evidence suggests patients consider a difference of $\geq 0.03$ points as important. ${ }^{18}$ We evaluated self reported function by having participants indicate at each follow-up visit, on a scale from $0 \%$ to $100 \%$, what their overall level of functioning was, with $0 \%$ representing no ability to function and 100\% representing their level of functioning before injury.

\section{Sample size}

The smallest difference in score SF-36 PCS that would be important for patients is not well established, and investigators have provided different estimates; however, a 3-5 point change in score on a 0-100 scale is often cited as a minimally important difference, based on the work by Stewart and colleagues. ${ }^{19}$ Based on our previous study of health related quality of life in patients with distal tibial fractures, ${ }^{15}$ and the TRUST pilot study, ${ }^{12}$ we anticipated a standard deviation of $\leq 12$ for SF-36 PCS scores. Considering these findings, we estimated that 250 patients per group would be required to have a power of $80 \%(\alpha=0.05$, two tailed) to identify a difference in absolute scores of 3 points between treatment and control groups. Our power calculation was based on detecting an average difference between the groups, rather than the test of the interaction term planned in the analysis, and was therefore conservative. Our sample size calculation is based on SF-36 PCS scores as our co-primary outcome measure, radiographic healing, is a surrogate for functional recovery.

\section{Interim analysis and guidelines for study discontinuation}

We planned an interim analysis when 300 patients had completed the trial, with review by a data monitoring committee using the O'Brien-Fleming stopping rule. ${ }^{20}$ The industry sponsor, however, conducted an unplanned interim analysis of blinded data from 237 TRUST patients with one year follow-up in November 2012 (groups were analyzed as "group A" and “group B”). Based on the finding of no difference in treatment effect between groups, and thus a conclusion of futility, the sponsor discontinued the study. We continued to collect data from patients up to March 2013. Submission was 
delayed as we dealt with the industry sponsor's many concerns, including requests for many post hoc subgroup analyses. All patients, clinicians, investigators, data analysts, and the industry sponsor remained blinded to allocation until analysis of all data was completed.

\section{Statistical analyses}

All patients enrolled were analyzed according to their randomized group, regardless of compliance with treatment or any other deviation from protocol (intention to treat principle). All patients without full follow-up, regardless of reason, were censored at their last follow-up. Data analyses were conducted with SAS version 9.4 (SAS Institute) with a threshold $\mathrm{P} \leq 0.05$. We calculated compliance with treatment by dividing the number of days that patients administered $\geq 18$ minutes of LIPUS/day from the day they received their study device to when they achieved radiographic healing or their 52-week follow-up, by the total number of days within this time period.

Primary analyses considered only available data without imputation for missing data. We used multilevel linear models to examine SF-36 PCS and scores on the health utilities index over time including time, treatment, the interaction between time and treatment, severity of soft tissue injury (open versus closed), and the interaction between severity and time as independent variables in the model. The multi-level model, which allows for the clustering of repeated measures within patients and the clustering of patients within treatment site, included three levels: site, patient, and follow-up visit. We also performed adjusted analyses that added the following independent variables to our models: age, sex, smoking status, fracture gap, fracture pattern, and fracture grade, but not severity or its interaction with time because open versus closed is part of

\begin{tabular}{|c|c|}
\hline \multicolumn{2}{|c|}{ Assessed for eligibility $(n=3105)$} \\
\hline & $\begin{array}{l}\text { Excluded }(n=2604): \\
\quad \text { Did not meet eligibility criteria }(n=2506) \\
\text { Patients refused to participate }(n=98)\end{array}$ \\
\hline \multicolumn{2}{|c|}{ Randomized $(n=501)$} \\
\hline$\sqrt{2}$ & 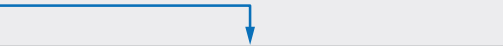 \\
\hline Assigned to treatment (active LIPUS) $(n=250)$ & Assigned to control (sham LIPUS) $(n=251)$ \\
\hline$\downarrow$ & $\frac{1}{1}$ \\
\hline $\begin{array}{l}\text { Timing of loss to follow-up: } \\
\text { Patients provided no data after discharge* } \\
(n=10) \\
\text { Patients were lost before } 6 \text { months }(n=28) \\
\text { Patients were lost after } 6 \text { months }(n=19)\end{array}$ & $\begin{array}{l}\text { Timing of loss to follow-up: } \\
\text { Patients provided no data after discharge } \\
(n=10) \\
\text { Patients were lost before } 6 \text { months }(n=41) \\
\text { Patients were lost after } 6 \text { months }(n=11)\end{array}$ \\
\hline $\begin{array}{l}\text { Reason for loss to follow-up: } \\
\text { Withdrew consent }(n=19) \\
\text { Lost contact }(n=26) \\
\text { Other withdrawal }(n=10)\end{array}$ & $\begin{array}{l}\text { Reason for loss to follow-up: } \\
\text { Withdrew consent }(n=26) \\
\text { Lost contact }(n=28) \\
\text { Other withdrawal }(n=8)\end{array}$ \\
\hline$\downarrow$ & $\downarrow$ \\
\hline $\begin{array}{l}\text { Included in intention to treat analysis } \\
\text { of SF-36 PCS scores }(n=240) t\end{array}$ & $\begin{array}{l}\text { Included in intention to treat analysis } \\
\text { of SF-36 PCS scores }(n=241) \dagger\end{array}$ \\
\hline
\end{tabular}

Fig 1 | Recruitment of patients with tibial fractures and follow-up schedule the fracture grade variable. We conducted a sensitivity analysis for SF-36 PCS scores using non-linear multiple imputation (fully conditional specification approach) to deal with missing observations. ${ }^{2122}$

We evaluated time to radiographic healing, time to full weight bearing, time to return to work without restrictions, time to return to household duties without restrictions, time to return to at least $80 \%$ of function before injury, and time to return to leisure activities without restrictions, in LIPUS compared with control groups, using Cox proportional hazards models stratified by severity of soft tissue injury and clinical site. For time to radiographic healing, we also performed an adjusted Cox proportional hazards model including age, sex, smoking status, fracture gap, fracture pattern, and fracture grade as covariates. This model was not stratified by severity of soft tissue injury because open/ closed is included in the fracture grade variable. The date on which patients received their study device was used as the starting point for all time to event analyses. In our analyses of binary endpoints, because they would be missing completely at random (uninformed censoring), we included patients who died or who did not continue to one year because the trial was stopped early. Data from patients lost to follow-up were also included up to the point of loss (informed censoring).

We compared the proportion of adverse events related to the device, deaths, unplanned secondary procedures related to bone healing and infection, and the occurrence of non-union between ultrasound and control groups with Pearson's $\chi^{2}$ test or Fisher's exact test.

\section{Subgroup and sensitivity analyses}

We planned one a priori subgroup analysis based on open versus closed fractures, with the anticipation that open fractures would show larger treatment effects.

\section{Patient involvement}

No patients were involved in setting the research question or the outcome measures, nor were they involved in developing plans for recruitment, design or implementation of the study. We did, however, respond to patient feedback regarding excessive questionnaire burden in the TRUST pilot study. ${ }^{12}$ No patients were asked to advise on interpretation or writing up of results. There are no plans to disseminate the results of the research to study participants or the relevant patient community.

\section{Results}

\section{Participants}

Of 3105 patients screened, 501 met eligibility criteria, provided informed consent, and were randomized (fig 1). Most exclusions of potentially eligible patients occurred because fractures were not amenable to an intramedullary nail, patients were aged $<18$, patients stated they could not comply with the study protocol, or because there was concomitant injury that was likely to impair function for longer than their tibial fracture.

Most patients were men who had been injured in a fall resulting in a closed tibial fracture, and 368 (74\%) were employed before their injury; patients' 
characteristics were similar in intervention and control groups (table 1). As a result of the industry sponsor's decision to stop the study early, 73 patients were followed up for fewer than 12 months (see table A in appendix for further details). Data on patient compliance were available for 424 patients (62 did not return their study device, and 15 devices failed to record compliance); 189 (45\%) had $\geq 75 \%$ compliance and 119 (28\%) had $\geq 50 \%$ but $<75 \%$ compliance. There was no difference in compliance between treatment groups.

We acquired SF-36 PCS data from 481/501 (96\%) patients, from whom we had 2303/2886 (80\%) observations. We included data on radiographic healing for

\begin{tabular}{|c|c|c|c|}
\hline & $\begin{array}{l}\text { LIPUS } \\
(n=250)\end{array}$ & $\begin{array}{l}\text { Sham } \\
(n=251)\end{array}$ & $\begin{array}{l}\text { Total } \\
(\mathrm{n}=501)\end{array}$ \\
\hline Mean (SD) age (years) & $37.1(13.2)$ & $39.1(14.6)$ & $38.1(13.9)$ \\
\hline Women & $81(32)$ & $75(30)$ & $156(31)$ \\
\hline Men & $169(68)$ & $176(70)$ & $345(69)$ \\
\hline Employed before injury & $184(74)$ & $184(73)$ & $368(74)$ \\
\hline \multicolumn{4}{|l|}{ Mechanism of injury: } \\
\hline Motor vehicle crash & $25(10)$ & $14(6)$ & $39(8)$ \\
\hline Motor vehicle crash (pedestrian) & $28(11)$ & $30(12)$ & $58(12)$ \\
\hline Motorcycle crash & $25(10)$ & $35(14)$ & $60(12)$ \\
\hline Crush injury & $7(3)$ & $5(2)$ & $12(2)$ \\
\hline Fall & $84(34)$ & $87(35)$ & $171(34)$ \\
\hline Twist & $25(10)$ & $20(8)$ & $45(9)$ \\
\hline Direct trauma (penetrating) & 0 & $4(2)$ & $4(18)$ \\
\hline Direct trauma (blunt) & $43(17)$ & $33(13)$ & $76(15)$ \\
\hline Other & $13(5)$ & $23(9)$ & $36(7)$ \\
\hline Current smoker & $79(32)$ & $86(34)$ & $165(33)$ \\
\hline \multicolumn{4}{|l|}{ Diabetes*: } \\
\hline Any & $11(4)$ & $19(8)$ & $30(6)$ \\
\hline Insulin dependent & $7(3)$ & $8(3)$ & $15(3)$ \\
\hline Non-insulin dependent & $4(2)$ & $11(4)$ & $15(3)$ \\
\hline \multicolumn{4}{|l|}{ Fracture: } \\
\hline Open & $58(23)$ & $56(22)$ & $114(23)$ \\
\hline Closed & $192(77)$ & $195(78)$ & $387(77)$ \\
\hline \multicolumn{4}{|c|}{ Gustilo classification for open fractures (\% of all patients): } \\
\hline I & $26(10)$ & $25(10)$ & $51(10)$ \\
\hline$\|$ & $15(6)$ & $19(8)$ & $34(7)$ \\
\hline IIIA & $15(6)$ & $11(4)$ & $26(5)$ \\
\hline IIIB & $2(1)$ & $1(<1)$ & $3(1)$ \\
\hline \multicolumn{4}{|c|}{ Tscherne classification for closed fractures (\% of all patients): } \\
\hline 0 & $64(26)$ & $62(25)$ & $126(25)$ \\
\hline 1 & $110(44)$ & $110(44)$ & $220(44)$ \\
\hline 2 & $16(6)$ & $20(8)$ & $36(7)$ \\
\hline 3 & $2(1)$ & $3(1)$ & $5(1)$ \\
\hline \multicolumn{4}{|l|}{ Type of fracture*: } \\
\hline Comminuted & $57(23)$ & $67(27)$ & $124(25)$ \\
\hline Transverse & $64(26)$ & $55(22)$ & $119(24)$ \\
\hline Oblique & $77(31)$ & $77(31)$ & $154(31)$ \\
\hline Segmental & $6(2)$ & $2(1)$ & $8(2)$ \\
\hline Spiral & $82(33)$ & $95(38)$ & $177(35)$ \\
\hline \multicolumn{4}{|l|}{ Type of fixationt: } \\
\hline Nail with previous reaming & $249(100)$ & $249(99)$ & $498(>99)$ \\
\hline Nail without previous reaming & 0 & $2(1)$ & $2(<1)$ \\
\hline Adjudicated postoperative fracture gap $\ddagger$ & $10(4)$ & $5(2)$ & $15(3)$ \\
\hline
\end{tabular}

482/501 (96\%) patients in our analysis, of whom 82 were censored. We acquired health utilities index data from 481/501 (96\%) patients, from whom we had 2304/2886 (80\%) observations. Our rate of follow-up for secondary functional outcomes was 83\% (406/490) for return to at least $80 \%$ of function before injury, $78 \%$ (269/347) for return to work without limitations, 73\% (343/473) for return to household activities without limitations, 91\% (451/497) for return to full weight bearing, and $70 \%$ (321/457) for return to leisure activities without limitations. The rate of follow-up for secondary functional outcomes considered all enrolled patients minus those affected by early trial stoppage who had not yet experienced the event of interest.

\section{Effectiveness}

Results differed little between primary analyses, adjusted analyses, and analyses with imputation (fig A in appendix); we report here the unadjusted analyses. Our repeated measures analyses found no significant interaction for treatment by time $(\mathrm{P}=0.30$ for SF-36 PCS, fig 2; $\mathrm{P}=0.94$ for health utilities index). Treatment failed to influence SF-36 PCS scores (mean difference 0.55, $95 \%$ confidence interval -0.75 to $1.84 ; \mathrm{P}=0.41$; table $\mathrm{B}$ in appendix), or health utilities index scores (0.01, -0.02 to 0.05 ; $\mathrm{P}=0.44$; table $\mathrm{C}$ in appendix). Time to radiographic healing was also similar between groups (hazard ratio $1.07,95 \%$ confidence interval 0.86 to 1.34 ; $P=0.55$; fig 3; table D in appendix).

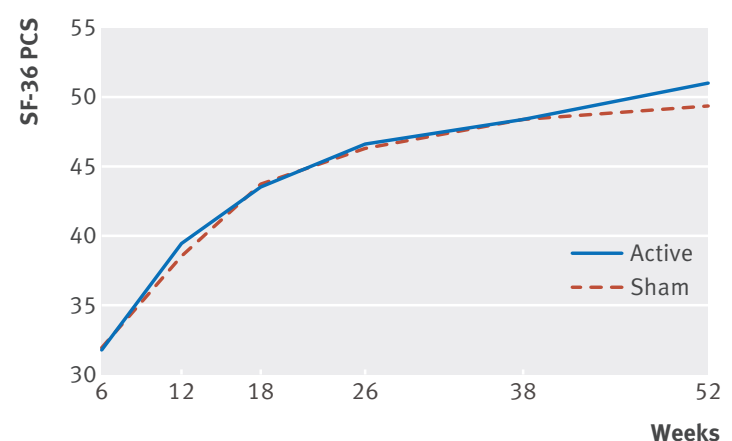

Fig 2 | SF-36 physical component summary (PCS) scores over time in patients with tibial fracture according to treatment with low intensity pulsed ultrasound or sham device

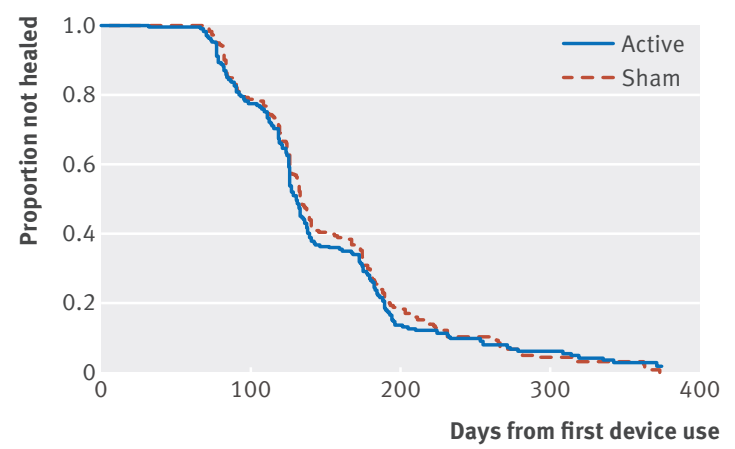

Fig 3 Kaplan-Meier time to event curve for radiographic fracture healing in patients with tibial fracture according to treatment with low intensity pulsed ultrasound or sham device 
We found no differences in time to return to work without limitations (hazard ratio 1.11, 95\% confidence interval 0.82 to $1.50 ; \mathrm{P}=0.51$; table $\mathrm{E}$ in appendix), time to return household activities without limitations ( 0.94 , 0.73 to 1.22 ; $\mathrm{P}=0.65$; table $\mathrm{F}$ in appendix), time to full weight bearing $(0.87 ; 0.70$ to 1.08 ; $P=0.21$; table $\mathrm{G}$ in appendix), time to return to $\geq 80 \%$ of function before injury (1.00, 0.80 to 1.25 ; $\mathrm{P}=0.97$; table $\mathrm{H}$ in appendix), or time to return to leisure activities without limitations (1.06, 0.77 to 1.46; $\mathrm{P}=0.72$; table I in appendix). Table 2 summarizes the results. Subgroup analyses suggested no difference in treatment effect for open versus closed fractures for SF-36 PCS scores ( $\mathrm{P}=0.59$ for interaction), health utilities index scores ( $\mathrm{P}=0.64$ for interaction), or radiographic healing ( $\mathrm{P}=0.65$ for interaction).

\section{Safety}

No site investigators reported adverse events associated with the study treatment. There was one death among the patients randomized to LIPUS and two among the patients randomized to sham treatment $(\mathrm{P}=0.62)$. The risks of unplanned secondary procedures related to bone healing (11/250 v 9/251; $\mathrm{P}=0.66)$, infection $(2 / 250 v$ $3 / 251 ; \mathrm{P}=1.00)$, and non-union (9/250 v 5/251; $\mathrm{P}=0.28)$ were similar between groups.

\section{Discussion}

\section{Principal findings}

In this randomized controlled clinical trial of patients undergoing surgery for fresh tibial fracture, the addition of low intensity pulsed ultrasound (LIPUS) did not improve functional recovery or accelerate radiographic healing.

\section{Strengths and limitations}

Strengths of this randomized trial include our pragmatic design; ${ }^{23}$ a sample size sufficient to generate narrow confidence intervals; multiple participating surgeons and centers, enhancing generalizability; strategies to reduce bias that included central randomization to ensure concealment, blinding of patients, clinicians, data collectors, outcome assessors, data analysts and the industry sponsor, and independent, blinded adjudication of eligibility and outcomes; and completion of $\geq 80 \%$ of all follow-up visits for our primary outcomes.

\begin{tabular}{|c|c|c|}
\hline Outcome measure & LIPUS & Sham \\
\hline Mean (SD) SF-36 PCS score & $51.0(7.9)$ & $49.3(8.8)$ \\
\hline Mean (SD) HUI-3 & $0.85(0.19)$ & $0.84(0.19)$ \\
\hline Radiographic healing $\dagger$ & $142 / 214(66)$ & $125 / 201(62)$ \\
\hline Return to work without limitations (368 worked before injury) & $126 / 149(85)$ & $110 / 138(80)$ \\
\hline Return to household activities without limitations & $157 / 190(83)$ & $147 / 185(80)$ \\
\hline Return to full weight bearing $\dagger$ & $188 / 234(80)$ & $194 / 229(85)$ \\
\hline Return to $\geq 80 \%$ of function before injury $\dagger$ & $156 / 221(71)$ & $148 / 208(71)$ \\
\hline Return to leisure activities without limitations & $99 / 175(57)$ & $95 / 166(57)$ \\
\hline
\end{tabular}

The primary limitation of our trial was our failure to obtain $100 \%$ follow-up for our primary outcomes, and larger loss to follow-up for some secondary outcomes; however, multiple imputation led to similar estimates of treatment effects, providing reassurance that loss to follow-up was unlikely to have biased our results.

Patient compliance was moderate and is a possible explanation for differences in results between TRUST and previous studies. Two previous trials of LIPUS for fracture healing ensured close to perfect compliance by having investigators administer LIPUS to patients in hospital ${ }^{24}$ or to officers stationed at a naval academy that were required to report for daily treatment. ${ }^{25}$ The former found no effect on time to when the callus was considered strong enough for safe removal of the fixator after high tibial osteotomy ( 0.8 weeks earlier, $95 \%$ confidence interval 2.3 weeks earlier to 0.71 weeks later; $\mathrm{P}=0.31),{ }^{24}$ and the latter found no effect on time to return to active duty after tibial stress fracture (0.4 days earlier, 14.1 days earlier to 13.3 days later; $\mathrm{P}=0.96){ }^{25}$

In the outpatient setting in which LIPUS is used, patients use the device themselves. Compliance with treatments is, in general, limited; ${ }^{26}$ this is particularly true of treatments that involve the level of inconvenience or burden associated with LIPUS (for example, the ninth edition of the American College of Chest Physicians Evidence-Based Clinical Practice Guidelines noted that compliance is the greatest challenge associated with use of outpatient limb compression devices in orthopedic surgical patients ${ }^{27}$ ). Thus, the compliance found in our trial-only $73 \%$ of our patients administered $\geq 50 \%$ of all recommended treatments-probably reflects patients' use in real clinical settings. The level of compliance we observed is high enough that, if effects on time to radiographic healing seen in previous studies were present in our patients, we would have seen a substantial (albeit possibly attenuated) impact of the device.

\section{Implications}

The FDA approved LIPUS for fracture healing in 1994 on the basis of small trials at high risk of bias that showed that LIPUS accelerated radiographic healing. ${ }^{28}$ Many medical devices, however, are approved for sale without randomized trial evidence of important benefit to patients. ${ }^{29}$ A study published in 2016 found that of 99 medical devices recently approved by the FDA, 43 were cleared or approved before a clinical study was published. ${ }^{30}$ Further, as is the case with LIPUS, ${ }^{31-33}$ device inventors or industry employees are often investigators on clinical trials that are used to gain regulatory approval. ${ }^{34}$ Our experience suggests the high desirability of demanding evidence from randomized trials conducted by investigators other than those who will gain financially from clinical use of the device before approval by regulatory agencies.

\section{Conclusions}

Among patients undergoing intramedullary nailing for a tibial shaft fracture the addition of low intensity pulsed ultrasound does not improve functional recovery or accelerate radiographic healing. 
Contributors: JWB (chair of writing group), MB (associate chair), TAE, ES, IDH, PT III, K-SL, and GHG conceived and designed the study. JWB, MB, and GHG acquired the funding. Data collection was monitored by Alquest (contract research organization). JWB, DH-A, SM-K, and GHG developed the statistical analysis plan, conducted the analysis and interpreted the data. JWB, DH-A, and SM-K had full access to all the data in the study and take responsibility for the integrity of the data and the accuracy of the data analysis. JWB drafted the manuscript, which was revised by the other authors (MB, TAE, ES, JDH, PT III, K-SL, GHG, GJDR and CBJ). All authors read and approved the final manuscript. JWB is guarantor.

Funding: This study was an investigator-initiated trial, supported by grants from the Canadian Institutes of Health Research (CIHR) (MCT 67815, Co-Pls: GH Guyatt, M Bhandari), and an industry grant from Smith \& Nephew. The CIHR had no role in the design and conduct of the study; collection, management, analysis, and interpretation of the data; preparation, review, or approval of the manuscript; or decision to submit the manuscript for publication. Smith \& Nephew personnel reviewed initial drafts of the trial protocol and raised many issues about alternative approaches to study design. Issues regarding the protocol were resolved through negotiation between Smith \& Nephew and the trial steering committee. Final decisions regarding the protocol and issues that arose during the conduct of the trial were the purview of the trial steering committee. The investigators had full access to all trial data. Smith \& Nephew had no role the initial preparation of the current study manuscript but had the right to review the manuscript and make non-binding comments and suggestions.

Competing interests: All authors have completed the ICMJE uniform disclosure form at www.icmje.org/coi_disclosure.pdf and declare: TAE, $E S$, and MB have received consulting fees from Smith \& Nephew, the manufacturer of the study device. PT receives royalties from Smith \& Nephew. GJDR is a paid consultant for Bioventus LLC, which is $51 \%$ owned by Essex Woodlands and 49\% by Smith \& Nephew. MB is supported, in part, by a Canada research chair, McMaster University. Ethical approval: The study protocol was approved by McMaster University research ethics board (REB\#08-171) and local boards at each participating site.

Data sharing: Full dataset and statistical code is available from the corresponding author. Patient consent was not obtained for data sharing, but the data are anonymized and risk of identification is low.

Transparency: The lead author affirms that the manuscript is an honest, accurate, and transparent account of the study being reported; that no important aspects of the study have been omitted; and that any discrepancies from the study as planned have been explained.

This is an Open Access article distributed in accordance with the Creative Commons Attribution Non Commercial (CC BY-NC 3.0) license, which permits others to distribute, remix, adapt, build upon this work non-commercially, and license their derivative works on different terms, provided the original work is properly cited and the use is noncommercial. See: http://creativecommons.org/licenses/by-nc/3.0/.

1 Heckman JD, Sarasohn-Kahn J. The economics of treating tibia fractures. The cost of delayed unions. Bull Hosp Jt Dis 1997; $56: 63-72$

2 Busse JW, Morton E, Lacchetti C, Guyatt GH, Bhandari M. Current management of tibial shaft fractures: a survey of 450 Canadian orthopedic trauma surgeons. Acta Orthop 2008;79:689-94. doi:10.1080/17453670810016722.

3 Sarmiento A, Sharpe FE, Ebramzadeh E, Normand P, Shankwiler J. Factors influencing the outcome of closed tibial fractures treated with functional bracing. Clin Orthop Relat Res 1995;(315):8-24

4 Wachovia Capital Markets. Equity research: bone growth stimulation 2008 outlook. 2007.

5 Griffin XL, Parsons N, Costa ML, Metcalfe D. Ultrasound and shockwave therapy for acute fractures in adults. Cochrane Database Syst Rev 2014;6:CD008579.

6 Busse JW, Kaur J, Mollon B, et al. Low intensity pulsed ultrasonography for fractures: systematic review of randomised controlled trials. BMJ 2009;338:b351. doi:10.1136/bmj.b351.

7 The worldwide orthopaedic market: 2004-2005. Knowledge Enterprises. The Ortho People. October, 2005. https://www. orthoworld.com/docs/pdf/world_orthomarket/wwo-2005.pdf

8 Cassel CK, Guest JA. Choosing wisely: helping physicians and patients make smart decisions about their care. JAMA 2012;307:1801-2. doi:10.1001/jama.2012.476.

9 Lenzer J. Unnecessary care: are doctors in denial and is profit driven healthcare to blame? BM/ 2012;345:e6230. doi:10.1136/bmi.e6230.

10 The BMJ's Too Much Medicine Campaign http://www.bmj.com/ too-much-medicine
11 Moynihan R, Glasziou P, Woloshin S, Schwartz L, Santa J, Godlee F. Winding back the harms of too much medicine. BMJ 2013;346:11271. doi:10.1136/bmj.f1271.

12 Busse JW, Bhandari M, Einhorn TA, et al. The TRUST Investigators. Trial to re-evaluate ultrasound in the treatment of tibial fractures (TRUST): a multicenter randomized pilot study. Trials 2014;15:206. doi:10.1186/1745-6215-15-206

13 McHorney CA, Ware JE Jr, Lu JF, Sherbourne CD. The MOS 36-item Short-Form Health Survey (SF-36): III. Tests of data quality, scaling assumptions, and reliability across diverse patient groups. Med Care 1994;32:40-66. doi:10.1097/00005650-199401000-00004.

14 Ware JE Jr, ed. SF-36 Physical \& Mental Health Summary Scales: A User's Manual. Quality Metric Inc, 1997.

15 Bhandari M, Sprague S, Hanson B, et al. Health-related quality of life following operative treatment of unstable ankle fractures: a prospective observational study. J Orthop Trauma 2004:18:338-45. doi:10.1097/00005131-200407000-00002.

16 Busse JW, Bhandari M, Guyatt GH, et al. SPRINT Investigators. Use of both Short Musculoskeletal Function Assessment questionnaire and Short Form-36 among tibial-fracture patients was redundant. / Clin Epidemiol 2009;62:1210-7. doi:10.1016/j.jclinepi.2009.01.014.

17 Kooistra BW, Dijkman BG, Busse JW, Sprague S, Schemitsch EH, Bhandari $\mathrm{M}$. The radiographic union scale in tibial fractures: reliability and validity. J Orthop Trauma 2010;24(Suppl 1):S81-6. doi:10.1097/ BOT.0b013e3181ca3fd1.

18 Horsman J, Furlong W, Feeny D, Torrance G. The Health Utilities Index (HUI): concepts, measurement properties and applications. Health Qual Life Outcomes 2003;1:54. doi:10.1186/1477-7525-1-54.

19 Stewart AL, Greenfield S, Hays RD, et al. Functional status and well-being of patients with chronic conditions. Results from the Medical Outcomes Study. JAMA 1989;262:907-13. doi:10.1001/ jama.1989.03430070055030.

20 O'Brien PC, Fleming TR. A multiple testing procedure for clinical trials. Biometrics 1979:35:549-56. doi:10.2307/2530245.

21 Carpenter J, Kenward M. Multiple Imputation and its Application.1st ed. Wiley, 2013. doi:10.1002/9781119942283.

22 Graham JW. Missing data analysis: making it work in the real world. Annu Rev Psychol 2009;60:549-76. doi:10.1146/annurev.psych.58.110405. 085530

23 Tunis SR, Stryer DB, Clancy CM. Practical clinical trials: increasing the value of clinical research for decision making in clinical and health policy. JAMA 2003;290:1624-32. doi:10.1001/jama.290.12. 1624.

24 Tsumaki N, Kakiuchi M, Sasaki J, Ochi T, Yoshikawa H. Low-intensity pulsed ultrasound accelerates maturation of callus in patients treated with opening-wedge high tibial osteotomy by hemicallotasis. J Bone Joint Surg Am 2004;86-A:2399-405.

25 Rue JP, Armstrong DW 3rd, Frassica FJ, Deafenbaugh M, Wilckens JH. The effect of pulsed ultrasound in the treatment of tibial stress fractures. Orthopedics 2004;27:1192-5.

26 Osterberg L, Blaschke T. Adherence to medication. N Engl / Med 2005;353:487-97. doi:10.1056/NEJMra050100.

27 Falck-Ytter Y, Francis CW, Johanson NA, et al. American College of Chest Physicians. Prevention of VTE in orthopedic surgery patients: Antithrombotic Therapy and Prevention of Thrombosis, 9th ed: American College of Chest Physicians Evidence-Based Clinical Practice Guidelines. Chest 2012;141(Suppl):e278S-325S. doi:10.1378/chest.11-2404.

28 Rubin C, Bolander M, Ryaby IP, Hadjiargyrou M. The use of low-intensity ultrasound to accelerate the healing of fractures. / Bone Joint Surg Am 2001;83-A:259-70.

29 Thompson M, Heneghan C, Billingsley M, Cohen D. Medical device recalls and transparency in the UK. BMJ 2011;342:d2973. doi:10.1136/bmj.d2973.

30 Marcus HJ, Payne CJ, Hughes-Hallett A, et al. Regulatory approval of new medical devices: cross sectional study. BM/ 2016:353:12587. doi:10.1136/bmj.i2587.

31 In Memoriam-John Ryaby. The Bioelectromagnetics Society. https:// www.bems.org/node/483

32 Heckman JD, Ryaby JP, McCabe J, Frey JJ, Kilcoyne RF. Acceleration of tibial fracture-healing by non-invasive, low-intensity pulsed ultrasound. J Bone Joint Surg Am 1994;76:26-34

33 Kristiansen TK, Ryaby JP, McCabe J, Frey JJ, Roe LR. Accelerated healing of distal radial fractures with the use of specific, low-intensity ultrasound. A multicenter, prospective, randomized, double-blind, placebo-controlled study. J Bone Joint Surg Am 1997:79:961-73.

34 Wilmshurst P. The regulation of medical devices. BMJ 2011;342: d2822. doi:10.1136/bmj.d2822

Appendix: Supplementary material (committee members; supplementary fig A; supplementary tables A-I) 